\title{
Human Facial Age Estimation Using Whale MLP-NN
}

\author{
Abhishek Rajeshbhai Mehta \\ Assistant Professor, PICA Science, Gujarat, India \\ abhishekmehta3094@gmail.com
}

\begin{abstract}
Recently, from the facial images, the estimation of age automatically is a raising research area. Several studies have been conducted in the detection of age strategy, however, some studies only show better performance It is because of the great control of the external metrics namely lifestyle, environment, and numerous expression that are available in the face image. The main aim of this work is to present an MLP-NN with the WOA in order to detect the age from the face image. The developed Whale MLP-NN model is used to recognize the person's age in the image via the initial training with the facial features. In order to train the adopted optimization model, the features are offered using the AAM and scattering transform. Moreover, to detect the age, the optimal weights are exploited, and that is provided by the features with the Whale MLP-NN training. Finally, the experimental analysis exhibits that the developed optimization model is performed using the databases on the basis of measures namely AEO, MAE, and AEM.
\end{abstract}

Keywords: Age Estimation, Expression, Facial Image, MLP-NN, Optimization Algorithm, Optimal Weights

\begin{tabular}{ll} 
Nomenclature & \\
\hline Abbreviations & \multicolumn{1}{c}{ Descriptions } \\
\hline MLP-NN & Multilayer Perceptron Neural Network \\
AAM & Active Appearance Model \\
DNN & Deep Neural Network \\
WOA & Whale Optimization Algorithm \\
AEO & Accuracy of Error of One Age Category \\
CNN's & Convolutional Neural Networks \\
AEM & Accuracy of An Exact Match \\
AGEn & Age Group-n Encoding \\
ResNet & Deep Residual Network \\
LAD & Local Age Decoding \\
MAE & Mean Absolute Error \\
LSDML & Label-Sensitive Deep Metric Learning \\
FFNNs & Feed-Forward Neural Networks \\
\hline
\end{tabular}

\section{Introduction}

In human beings, the estimation of facial age is considered as one of the hot topics in the last decades. Currently, in many places, the cameras are strewn all over the globe and capture our facial expressions everywhere that we travel. Therefore, still, facial soft biometrics may be considered as the important present attribute that human beings rely on for many research areas. In diverse people, the aging process is considered different because normally people in similar age groups exhibit several facial appearances. Therefore, the estimation of facial age is considered as one of the important terrible tasks. Additionally, human has the ability to predict the identification, gender, expression and person race in an accurate way than predicting person age. Accordingly, numerous facial automatic age calculation techniques were modeled to overcome aforesaid issues.

For everyone, the facial aging procedure is occupied with arbitress as well as it is not stationary. In numerous points of view, randomness subsists namely environment, lifestyle, diverse diets, and several genes. Because of these factors, human aging is affected, and that leads to aging diverse in appearance. People of similar age in real work might somewhat appear different, such as younger or older while compared with each other. Conversely, due to the gradual and slow aging process, the faces from near 
ages look the same. Now and then it is very difficult to find who is younger or older amid two faces from close ages. Hence, there is a high correlation amid age classes, especially for nearer ages.

In current days, numerous methods were presented to enhance the facial age estimation performance that can be approximately categorized into two classifications such as age estimator based as well as face representation based. Nevertheless, face representation to the feature descriptor used in preceding techniques are generally hand crafted that might loss very important information and needs expert knowledge. In order to identify, the feature learning was presented that learns from raw pixels discriminative filters directly for healthy age-associated face representation. The handcrafted features show the minimum performance when comparing with those data-driven techniques which are not yet fulfilled in numerous applications due to easy linear filters which are not influential sufficient to use the non-linear relationship amid age labels as well as face samples especially while face example is captured in uncontrolled environments [11] [12]. To overcome these restrictions deep learning base approach was exploited to learn a hierarchal nonlinear mappings series through stacked DNN to transfer the given face images to the age label space. For instance, a multi-scale model was proposed to learn the deep face indications to forecast the age value to the supplementary gender as well as ethnicity information. An ordinal regression model was developed through DCNN to use the age series information. Nowadays, CNNs was exhibited few suitable performances in diverse fields of face associated analysis such as face recognition, face alignment, face verification, gender as well as age classification. CNN as well as deep learning was efficiently used to transfer the facial images to output age labels. This technique is diverse from handcrafted-based techniques that physically extract the features from the face. Nevertheless, numerous conventional CNN techniques are higher and highly complicated with high network parameters as well as layers, maximum training time and highly training dataset that brought pricey calculation cost as well as storage overhead.

The main objective of this work is to present a new age recognition model based on MLP-NN. Moreover, this work also adopts the WOA algorithm to find the age. Here, three main processes are involved such as feature components, and then the AAM, as well as scattering transform, is exploited. In the last phase, the aforesaid two features are exploited to find the age by the developed optimization algorithm.

\section{Literature Review}

In 2019, Zichang Tan et al [1], developed an AGEn technique to search the association amid the genuine age as well as its nearby ages. In this work, the nearby ages were clustered into similar groups, and every age corresponding to the " $n$ " groups. In the training phase, the independent class was created, in that the similar age group was grouped. Based on that, the unique age calculation issue was transferred into binary classification sub-issues series. In order to deal with the sub-issues, a DCNN with multi classifier was modeled. Furthermore, a LAD scheme was developed to present to speed up the prediction procedure. Moreover, to mitigate the inequity data learning issue of every classifier, to the unified objective model a penalty factor was inserted.

In 2019, Hongyu Yang et al [2], developed a new generative adversarial network on the basis of the technique. For basic subject-definite characteristics as well as age particular facial alters regarding the elapsed time it individually models. It assures that the modeled faces available preferred aging effects when custody personalized properties stable. Furthermore, concurrently train a single generator an adversarial learning model was developed as well as multiple discriminators ensuing in smooth incessant face gaining series.

In 2019, Kuan-Hsien Liu et al [3], modeled an automatic estimation of age technique to the human faces persists to have a significant part in pattern recognition as well as computer vision. Numerous researches in terms of estimation of face age were concentrated on two effects such as classification model and feature extraction. Away from the proposed model from the convention age estimation methods, a diverse effect was considered system structuring that was in a constrained circumstance presented a fixed learning model as well as fixed feature type, how to model a structure to enhance the performance of the age estimation on the basis of a constraint. Moreover, a four phase integration model was presented for face age estimation. This model initiates from the gender identification as well as subsequently passes to the next stage, the gender-specific age grouping as well as pursued using the $3^{\text {rd }}$ phase, estimation of age within age groups, and ultimately at the integration phase.

In 2020, OlatunbosunAgbo-Ajala and SerestinaViriri[4], worked on Deep learning with CNNs techniques was broadly exploited for these classification analyses. From human faces, it poses unrivaled benefits in discriminative image features extraction. Nevertheless, numerous conventional CNN-based techniques were modeled to be superior and larger with high complex a layer which creates it confronts to deploy with resource-constrained features. Hence, a lightweight CNN approach for lesser layers was 
modeled to calculate the apparent as well as real age of individuals which was deployed on mobile devices.

In 2018, Hao Liu et al [5], developed an LSDML technique for facial estimation of age. The developed LSDML aspires to look for a sequence of hierarchical nonlinear transformations to present face samples to a dormant general space, using ResNet whereas the face pair's similarities were equally isotonic to the age-diverse in a ranking conserving way. The developed model learns to excavate difficult meaningful samples as traversal access to total negative. Therefore, in the transformed space, the local manifold of face samples was conserved.

\section{Age Estimation using Developed Model}

In this section, the developed WOA-MLP-NN algorithm is portrayed. At first, from the database, the face image is subjected to the pre-processing phase. Subsequently, from the preprocessed image, the AAM features and the scattering are extracted. In the database, for each image, the feature vector comprises the AAM features as well as the scattering technique. Subsequently, by exploiting the feature vectors, the WOA-MLP-NN approach is trained. To the developed trained WOA MLP-NN classifier, the test image features are subjected as the input in the testing stage. The test image age is provided by the developed WOA-MLP NN approach. Fig 1 shows the diagrammatic representation of the proposed model.

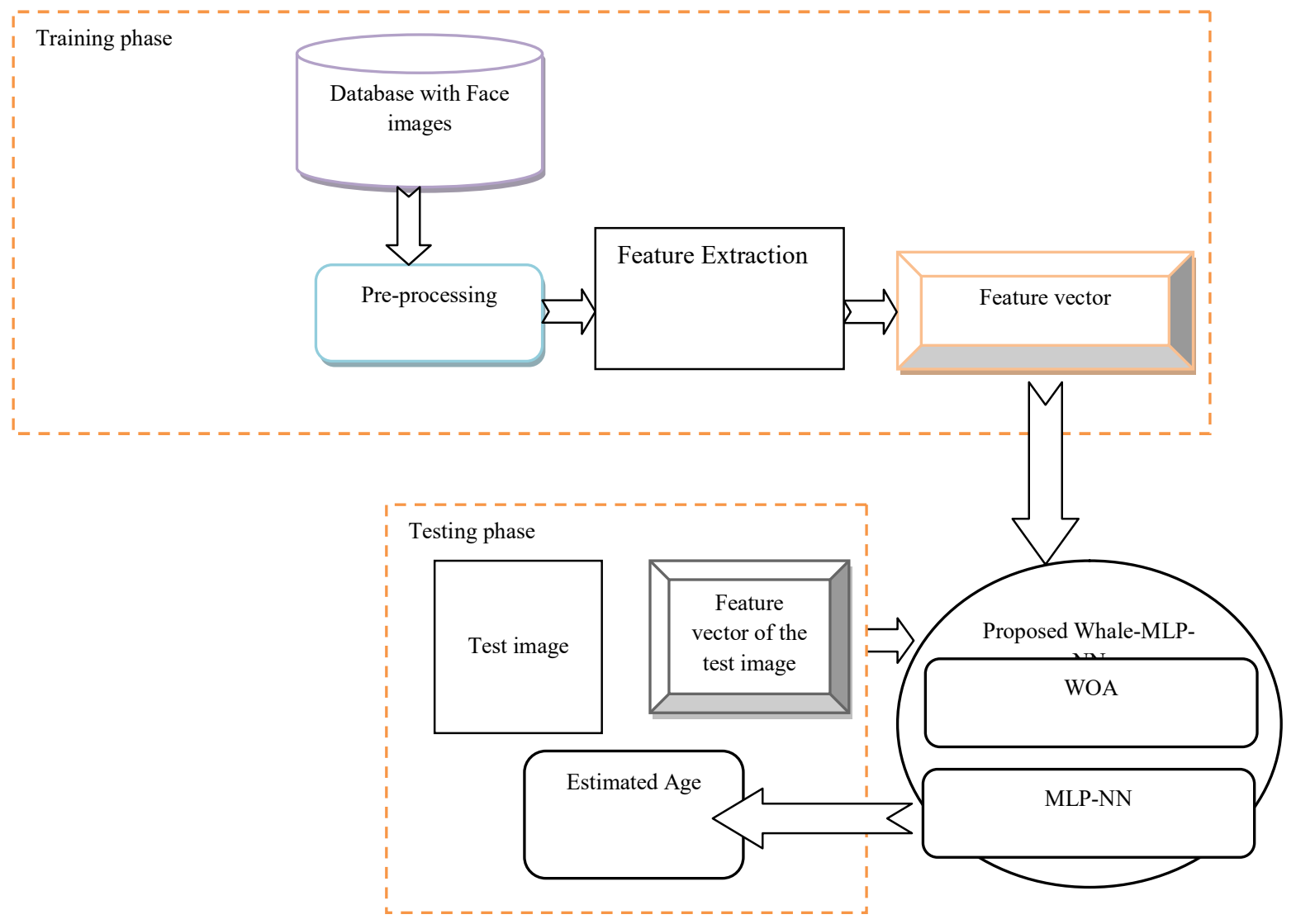

Fig 1. Schematic diagram of the proposed model

\subsection{Preprocessing}

Image preprocessing is considered the first stage of age detection. In the database, the developed method recognizes the age of each image. "N"count of persons face image is considered in the database for the experimentation. The main contribution of the developed model is to detect the "N" face image age. Eq. (1) indicates the formulation of the input database.

$$
\mathrm{D}=\left\{\mathrm{I}_{\mathrm{i}}\right\} \quad \mathbf{1} \leq \mathrm{i} \leq \mathrm{N}
$$

wherein, $I_{i}$ exhibits $\mathrm{i}^{\text {th }}$ face image in the database. In the database, the face image might possess numerous unnecessary areas. The face image preprocessing evades those areas which are present in the face images and presented the necessary face areas. The Viola-Jones object detection model is exploited in this paper to attain the face area from the image[6]. For the face detection process, the conventional 
Viola-Jones approach is particularly exploited to attain the face area appropriate for the feature extraction procedure.

\subsection{Feature Extraction on the basis of AAM as well as Scattering transforms technique}

For the age estimation, the developed technique extracts the needed features via the conventional techniques, AAM as well as scattering transform. The Morlet features of the images are learned by scattering transform with numerous orientations as well as scales. Hence, the scattering features aids in deep training networks. Both the texture as well as shape features of the face image are extracted by the AAM. Hence, a synthetic image is produced by means of texture as well as shape features. In the face images, the scattering features available are extracted using the scattering transform approach [7] and the active appearance is extracted via the conventional AAM [8].

\section{(i) AAM model}

By means of the conventional AAM model [8], the developed technique recognizes the features of active appearance for the image. In the image, by positioning the several feature points, the AAM approach identifies the shape features as well as the texture of the image. The image shape vector is identified by the AMM from the feature points ascertains via the semi or manual automatic way.

The ultimate synthesized image is stated as follows,

$$
\mathrm{I}_{\mathrm{S}}=\mathrm{W}_{\mathrm{X}_{\mathrm{S}}}^{-\mathbf{1}}\left(\mathrm{q}_{\mathrm{a}}^{\mathbf{1}}\left(\overline{\mathrm{a}}+\mathrm{E}_{\mathrm{a}} \mathrm{c}_{\mathrm{a}}\right)+\mathrm{q}_{\mathrm{a}}^{\mathbf{2}} \mathbf{1}\right)
$$

wherein, $E_{a}$ signifies the matrix with the gathering of the texture eigenvectors, $W_{X_{S}}^{-1}$ signifies the warping operation performed in the image, $\bar{a}$ signifies the mean texture, and $c_{a}$ signifies the counts of texture coefficients. By means of training of images, the synthesized images are attained from the database which is exploited to attain the target image. The target image indicates the solution that is attained via the normalized frame warping. The target image normalized texture as well as the synthesized image normalized texture possesses numerous differences that are called error. By computing the difference vector, the error amid the texture feature is minimized. Eq. (3) represents the difference between the target image texture feature as well as the synthesized image.

$$
\text { Error }=\| \text { Difference vector } \|^{2}
$$

\section{(ii) Scattering features}

For the age estimation, the subsequent feature exploited is the scattering feature. On the basis of the scattering approach, from the facial image, the scattering features are extracted. On the basis of ScatNet, the scattering features are extracted, a deep wavelet-based model [7]. In the input image, by using the averaging filter convolution the scattering features are extracted.

Let the image I scattering features comprise the series $C$. Therefore, a scattering wavelet transform network is modeled to find each coefficient-up to the series $\mathrm{m}$. The coefficients of higher-order scattering have enhanced the robustness rather than the lower order coefficients.

The scattering coefficients of the $C$ th layer formulation are stated in the eq. (4).

$$
\mathrm{K}_{\mathrm{C}} \mathrm{I}(\mathrm{m}, \wedge)=||\left|\mathrm{I} \otimes \beta_{\delta_{\mathbf{1}}}\right| \otimes \beta_{\delta_{\mathbf{2}}}|\cdots| \otimes \beta_{\delta_{\mathrm{i}}}|\ldots| \otimes \beta_{\delta_{\mathrm{m}}}\left|\otimes \eta_{\mathrm{L}}(\mathrm{m})\right|
$$

wherein, $\beta_{\delta_{1}}$ signifies first high-frequency variable of the image $\eta_{L}(m)$ signifies the averaging filter, $L$ signifies scaling factor, $I$ signifies the input image, and $\otimes$ signifies the convolution operator.

\section{(iii) Feature vector for training principle}

From the AAM as well as the scattering transform, the features need to be integrated jointly as a single feature vector to create the extracted features appropriate for the developed classifier training. The below expressions states the features from the AAM approach as well as the scattering transform.

$$
\begin{aligned}
& \mathrm{F}_{\mathrm{h}}(\mathrm{AAM})=\left\{\mathrm{a}_{\text {norm }}\left(\mathrm{I}_{\mathbf{1}}\right), \mathrm{a}_{\text {norm }}\left(\mathrm{I}_{\mathbf{2}}\right), \ldots \ldots \ldots \ldots \ldots \mathrm{a}_{\text {norm }}\left(\mathrm{I}_{\mathrm{N}}\right)\right\} \\
& \mathrm{F}_{\mathrm{g}}(\text { scatter })=\left\{\mathrm{K}_{\mathrm{C}}\left(\mathrm{I}_{\mathbf{1}}\right), \mathrm{K}_{\mathrm{C}}\left(\mathrm{I}_{\mathbf{2}}\right), \ldots \ldots \ldots \ldots \ldots \mathrm{K}_{\mathrm{C}}\left(\mathrm{I}_{\mathrm{N}}\right)\right\}
\end{aligned}
$$

wherein, $a_{\text {norm }}$ indicates normalized texture, $N$ indicates the number of images in the database $K_{C}$ indicates scattering coefficient. The developed technique integrated feature vector is indicated as follows:

$$
\text { Featurevector } F=\left[\begin{array}{ll}
F_{h}(\text { scatter }) & F_{h}(A A M)
\end{array}\right]
$$

wherein, $F_{h}$ (scatter) indicates features attained from the scattering transform approach. $F_{h}(A A M)$

indicates the face image features attained from the AAM approach. The feature vector total size is indicated as $1 \times k$, whereas $k$ indicates the number of features. 


\section{Developed Whale-MLP-NN for the Age Estimation}

\subsection{MLP-NN}

In the developed model, the subsequent phase is to find the age of the facial image which are available in the database. In this paper, the age detection model is developed and the Whale-MLP-NN model is proposed which integrates both the WOA and MLP-NN for the classification process.

Here, the MLP-NN and WOA method is exploited, the artificial NN is considered as the nonparametric and intelligent techniques, which is enthused by the biological nervous system.

The speedy developments by using the artificial neural network in the classification fields, regression, and pattern recognition have occurred.

FFNNs are a specific form of supervised NNs. It consists of processing elements set known as neurons. It is divided into various layers wherein each layer is entirely linked to the subsequent one. Moreover, the MLP model is stated as below:

The initial layer is the input layer which presents the network with the input layers; the ultimate layer is called output layers, and each layer amid the input as well as output layers is called hidden layers. In FFNNs, the generally used network is the MLP-NN [9]. In MLP, the neurons are interlinked in a one-directional as well as one-way model. Weights represent the link amid the neurons which are the actual numbers positioned in interval [1. 11]. In MLP, each layer is referred to as follows:

$$
\mathrm{o}_{\mathrm{i}}^{1}=\varphi\left(\mathrm{u}_{\mathrm{i}}^{1}\right)=\varphi\left(\sum_{\mathrm{j}=\mathbf{1}}^{\mathrm{n}_{1}} \mathrm{o}_{\mathrm{i}}^{1-\mathbf{1}_{\mathrm{w}}}{ }_{\mathrm{j}, \mathrm{i}}^{1}+\mathrm{w}_{\mathbf{0}, \mathrm{i}}^{1}\right), \mathbf{1} \leq 1 \leq \mathrm{L}
$$

wherein $\varphi($.$) indicates the layer activation function. Generally, it is configured$ as a nonlinear tangent hyperbolic function for in-between layers, that is in addition identified as hidden layers, as well as a linear function to produce the outcomes of the output layer. Index $l$ recognizes real layer in a network of $L$ non-input layers, $n l$ indicates the number of layer neurons, $l_{i}^{l}$ indicates the neuron output $i$ in the real layer $l, w_{j, i}^{l}$, $1 \leq l \leq n_{l-1} \quad$ indicates the weights linked with the links of neuron $i$ of layer $l$ with neurons of the previous layer $l-1$, and $w_{0, i}^{l}$ indicates the bias $i$ of the real layer. The layer output vector $l=0$ of length $n_{0}$ corresponds to the input characteristics vector, that is, $o^{(0)}=x$. Moreover, the ultimate layer output vector $l=L$ of length $n_{L}$, that is output layer of the network, corresponds to the network ensuing, (i.e.,) $o^{(L)}=y$.

\subsection{WOA model}

The WOA is a meta-heuristic approach it is enthused by the humpback whales hunting behavior that is called bubble-net hunting scheme [10]. Here, aforesaid whales exploit two models in order to search the prey location and then attacking it. At first, they encircle the prey, and finally, the bubble nets are created. In the optimization point perspective, while whales search for prey the exploration of search space is attained, and then at the time of attack behavior, the exploitation takes place. By exploiting the spiral movement, the In WOA, the bubble nets are simulated using a spiral movement. From actual humpback whales, this process mimics the helix-shaped movement process.

Conversely, a whale $x(t)$ possesses a location that can be updated by moving it in a spiral approximately the prey $x($ best $)$.

$$
\mathrm{x}(\mathrm{t}+\mathbf{1})=\mathrm{D} \cdot \mathrm{e}^{\mathrm{bl}} \cdot \cos (\mathbf{2 1})+\mathrm{x} \text { best }(\mathrm{t})
$$

wherein $l \in[-1,1]$ indicates an arbitrary number, $D=\mid x(t)$ - x best $(t) \mid$ indicates the distance amid $x(t)$ as well as $x($ best $)$ at iteration $t$, and $b$ indicates a constant variable exploited to explain the logarithmic spiral shape. By exploiting an encircling behavior the whales can update their locations on the basis of $x($ best $)$ as below:

$$
\begin{aligned}
& D=\left|C \cdot x_{\text {best }}(t)-x(t)\right| \\
& x(t+1)=x_{\text {best }}(t)-A . D \\
& C=\mathbf{2 r A}=\mathbf{2} \text { a.r }-\mathrm{a}
\end{aligned}
$$

The whales can concurrently swim around their prey beside a spiral-shaped path and through a shrinking circle is stated in Eq. (14)

$$
a=a-t \frac{a}{t_{\text {max }}}
$$




$$
\mathrm{x}(\mathrm{t}+\mathbf{1})=\left\{\begin{array}{l}
\mathrm{x}_{\text {best }}(\mathrm{t})-\mathrm{A} \text {.D if } \mathrm{r}_{\mathbf{1}}<\mathbf{0 . 5} \\
\text { D.e } \mathrm{e}^{\mathrm{bl}} \cdot \cos (\mathbf{2} \pi)+\mathrm{x}_{\text {best }}(\mathrm{t}) \text { if } \mathrm{r}_{\mathbf{1}}>\mathbf{0 . 5}
\end{array}\right.
$$

wherein $r 1 \in[0,1]$ indicates the selection probability of the approach of swimming around the prey. Nevertheless, humpback whales might be randomly searching for prey, and their location is updated on the basis of an arbitrarily chosen whale $x_{\text {rand }}(t)$ as below:

$$
\begin{aligned}
& D=\left|C \cdot x_{\text {rand }}(t)-x(t)\right| \\
& x(t+1)=x_{\text {rand }}(t)-\text { A.D }
\end{aligned}
$$

\subsection{WOA-MLP-NN model}

To train MLP-NN, the WOA approach is exploited in order to resolve a nonlinear regression. One of the common difficulty tasks a precise $\mathrm{NN}$ approach relies on training a $\mathrm{NN}$ to attain the optimal set of main biases as well as weights. The main drawbacks of conventional training approaches such as slow convergence speed as well as optima stagnation, hence the researchers motivate to search for a reliable solution to address aforesaid disadvantages. Therefore, by exploiting WOA the MLP optimizes to set the biases as well as weights of NN. The adopted technique exploits NN as a fitness function of the Whale algorithm to estimate its solution in the training phase. This analysis exploits the current solution as bias vectors and weight as well as transmits it to NN. On the basis of the NN prediction, the MSFE performance is computed. Until the maximum iteration is attained this case is repeated. The optimal solution is finally transmitted to the $\mathrm{NN}$ as a bias as well as weight vectors for usage in the testing phase.

In the testing stage, test image $I_{\text {test }}$, feature vector are given to the adopted optimization approach. On the basis of the trained optimal weights from the training stage, the Whale-MLP NN approach presents the age of the test image $I_{\text {test }}$.

\section{Experimental Procedure}

In this section, the experimental analysis of the developed Whale-MLP-NN model was compared with the conventional models for the age estimation from the facial image. The analysis measures like AEO, MAE, and AEM metrics the developed model performance. Moreover, the analysis techniques were taken into consideration for the analysis of the developed model outcomes. The evaluation of the developed model was experienced by exploiting 4 databases.

Fig 2 (a), (b), and (c)exhibit the evaluation of developed models with the conventional techniques for three metrics such as AEO, MAE as well as AEM. Here, 4 databases are exploited; the proposed model has a maximum performance over the conventional models. Fig 2 (a) shows the analysis of the proposed method regarding the MAE. Here, the proposed is $12 \%$ better than the conventional NN, $12 \%$ better than the conventional NN, $12 \%$ better than the conventional NN, $12 \%$ better than the conventional NN, $21 \%$ better than the conventional ANN, 14\% better than the conventional MLP, $10 \%$ better than the conventional KNN, $21 \%$ better than the conventional DBN, $21 \%$ better than the conventional NN, $20 \%$ better than the conventional SVM for IMBD. Fig 2 (b) shows the analysis of the proposed method regarding the AEO. In this fig, the proposed is $16 \%$ better than the conventional NN, $10 \%$ better than the conventional ANN, 9\% better than the conventional MLP, 8\% better than the conventional KNN, 7\% better than the conventional DBN, 7\% better than the conventional SVM for AEO. Fig 2 (c) exhibits the analysis of the proposed method regarding the AEM. 


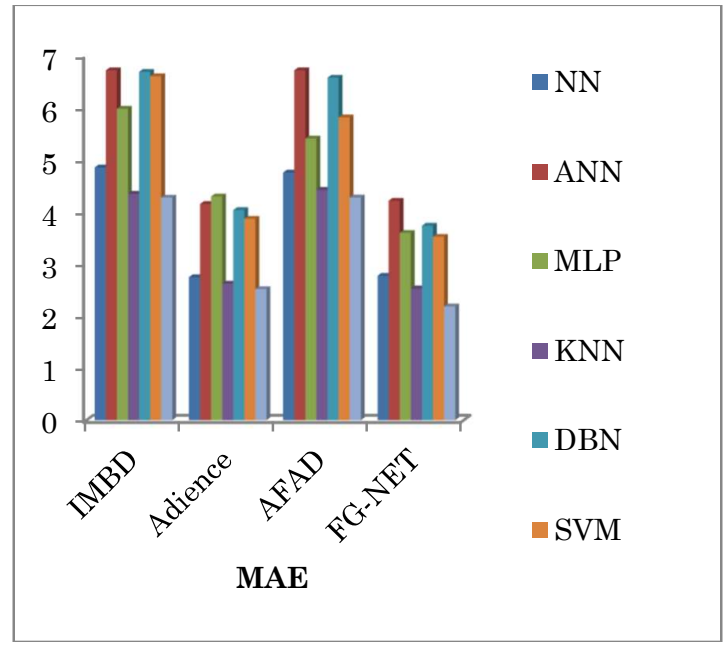

(a)

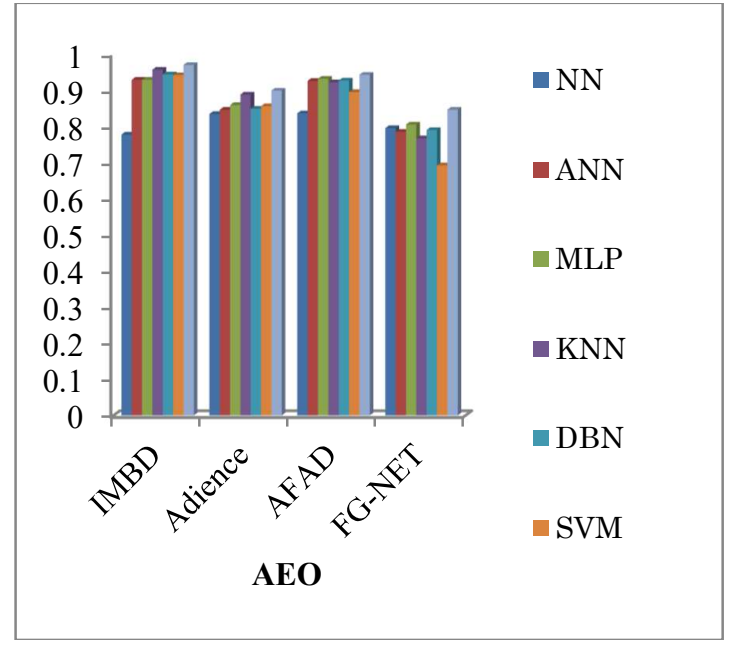

(b)

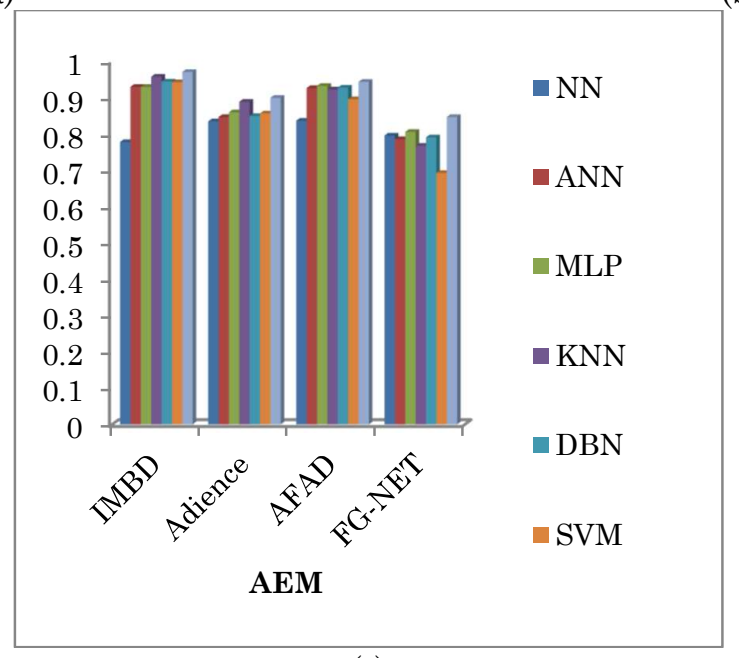

(c)

Fig 2 Performance analysis of developed technique over conventional techniques for the diverse database (a) MAE (b) $A E O$ (c) $A E M$

\section{Conclusion}

On the basis of the human face age estimation remnants an important issue in numerous fields. To calculate an age group or accurate age of a facial image, many of the conventional approaches need anenormous face data set attached with age labels. This imposes a constraint on the consumption of the enormously unlabeled or imperceptibly labeled training data, e.g., the enormous number of human photos in the social networks. These images might present no age label, however it was simple to gain the age difference for an image pair of the similar person. To enhance the accuracy of the age estimation, this work presents the Whale MLP-NN technique was adopted by integrating the idea of both the WOA and MLP-NN approaches. It was exploited to find the age via facial expression. In the developed model, each layer bias, as well as weights, was updated by exploiting the WOA. For the training, the age features of the developed optimization model were attained from both the AAM and scattering transform. Finally, the experimentation analysis was performed on the basis of the four databases such as AFAD database, Adience database, IMDB database, as well as FG-NET database based on several measures namely, AEO, MAE, and AEM. The overall analysis exhibits the superiority of the proposed model over the conventional models regarding age detection.

\section{Compliance with Ethical Standards}

Conflicts of interest: Authors declared that they have no conflict of interest.

Human participants: The conducted research follows the ethical standards and the authors ensured that they have not conducted any studies with human participants or animals. 


\section{References}

[1] Z. Tan, J. Wan, Z. Lei, R. Zhi, G. Guo and S. Z. Li, "Efficient Group-n Encoding and Decoding for Facial Age Estimation," in IEEE Transactions on Pattern Analysis and Machine Intelligence, vol. 40, no. 11, pp. 2610-2623, 1 Nov. 2018.

[2] H. Yang, D. Huang, Y. Wang and A. K. Jain, "Learning Continuous Face Age Progression: A Pyramid of GANs," in IEEE Transactions on Pattern Analysis and Machine Intelligence, vol. 43, no. 2, pp. 499-515, 1 Feb. 2021.

[3] K. -H. Liu and T. -J. Liu, "A Structure-Based Human Facial Age Estimation Framework Under a Constrained Condition," in IEEE Transactions on Image Processing, vol. 28, no. 10, pp. 5187-5200, Oct. 2019.

[4] O. Agbo-Ajala and S. Viriri, "A Lightweight Convolutional Neural Network for Real and Apparent Age Estimation in Unconstrained Face Images," in IEEE Access, vol. 8, pp. 162800-162808, 2020.

[5] H. Liu, J. Lu, J. Feng and J. Zhou, "Label-Sensitive Deep Metric Learning for Facial Age Estimation," in IEEE Transactions on Information Forensics and Security, vol. 13, no. 2, pp. 292-305, Feb. 2018.

[6] Vaishnavi, P.K and Pavitra, M.: 'Face Recognition using VIOLA-JONES Algorithm,' International Journal of Innovative Science and Research Technology, vol. 3, no. 2, pp. 163-167, 2018.

[7] Pandey, Prateekshit, Richa Singh, and Mayank Vatsa. "Face recognition using scattering wavelet under Illicit Drug Abuse variations." International Conference on Biometrics (ICB), Halmstad, pp. 1-6, 2016.

[8] Aziz Umit Batur and Monson H. Hayes, "Adaptive Active Appearance Models," IEEE transactions on image processing, vol. 14, no. 11, pp, 1707-1721, November 2005.

[9] R. AkkayaA. A. KulaksızÖ. Aydoğdu,"DSP implementation of a PV system with GA-MLP-NN based MPPT controller supplying BLDC motor drive", Energy Conversion and Management,vol.38, no, 8, pp.210-218, January 2007.

[10] Hailun WangFei WuLu Zhang,"Application of variational mode decomposition optimized with improved whale optimization algorithm in bearing failure diagnosis", Alexandria Engineering Journal, vol. 60, no. 5, pp. 4689-,5 April 2021.

[11] Priya M Shelke,Rajesh S Prasad,"Improved Sine-Cosine Algorithm For Anti Forensics JPEG Compression",Multimedia Research ,vol. 3, no. 1, January 2020.

[12] Nipanikar S I,Hima Deepthi V,"Enhanced Whale Optimization Algorithm and Wavelet Transform for Image Steganography", Multimedia Research, vol. 2, no. 3, July 2019. 